Editorial

\title{
Mutations and cancer genesis highlights on BRCAI and BRCA2 genes
}

\section{Editorial}

In humans, genes are located on 23 pairs of long strands of DNA called chromosomes. Changes to these genes, called mutations, play an important role in the development of cancer.

Most cancers start because of gene mutations that happen sometime during a person's lifetime. These are random events that sometimes happen inside a cell, without an obvious cause and generate what is called acquired mutations that only affect the cells that grow from the mutated cell.

Some other gene changes that lead to cancer may be inherited from a parent. Only about $5 \%$ to $10 \%$ of all cancers are thought to be related to an inherited gene change. Inherited mutations, are in every cell in the body, even the cells without cancer.

We also know that specific ethnic backgrounds are associated with a higher prevalence of mutations. These are known as founder mutations. BRCA1 and BRCA2 are human genes that produce tumor suppressor proteins. These proteins help repair damaged DNA. About $12 \%$ of women in the general population will develop breast cancer sometime during their lives and about $1.4 \%$ of women in the general population will develop ovarian cancer sometime during their lives. A woman's risk of developing breast and/or ovarian cancer is greatly increased if she inherits a deleterious mutation in the BRCA1 gene or the BRCA2 gene. Men with these mutations also have an increased risk of breast cancer, and both men and women who have harmful BRCA1 or BRCA2 mutations may be at increased risk of additional types of cancer.

Together, BRCA1 and BRCA2 mutations account for about 20 to 25 percent of hereditary breast cancers and about 5 to 10 percent of all breast cancers. In addition, mutations in BRCA1 and BRCA2 account for around 15 percent of ovarian cancers overall. Each child of a parent who carries a mutation in one of these genes has 50 percent chance of inheriting the mutation.

Deleterious mutations in BRCA1 and BRCA2 increase the risk of several cancers in addition to breast and ovarian cancer. BRCA1 mutations may increase a woman's risk of developing fallopian tube cancer and peritoneal cancer. Men with harmful BRCA1 or BRCA2 mutations have a higher risk of prostate cancer. Men and women with BRCA1 or BRCA2 mutations may be at increased risk of pancreatic cancer. Whom should undergo a BRCA mutation test?

Harmful BRCA1 and BRCA2 gene mutations are relatively rare in the general population. Therefore, most experts agree that mutation testing should always take into account several factors, including family history, ethnicity and personal history of either breast or ovarian cancer. In terms of family history, the latter should suggest the possible presence of a harmful mutation in BRCA1 or BRCA2, means, a diagnosis of breast or ovarian cancer at a young age, also women with a history of breast cancer twice in their own lifetime, women with relatives in first degree of separation with a history of both ovarian and breast cancers, males with breast cancer, as well as
Volume 2 Issue 6 - 2015

\author{
Hajj Adel Anis \\ Cedars - Jebel Ali International Hospital, UAE
}

Correspondence: Haji Adel Anis, Medical Oncologist at Cedars - Jebel Ali International Hospital, 9370 Rue Lajeunesse, Montreal, UAE, Tel 438-992-55I6, Email ahajj@dr.com

Received: July 17, 2015 | Published: July 20, 2015

multiple affected relatives with early onset breast or ovarian cancer. Finally, some data show that it would be probably reasonable to test even if family history is of limited informative background.

Mutations in BRCA1 and BRCA2 are more common in certain racial/ethnic populations. People of Ashkenazi jewish decent have a prevalence of harmful BRCA1 and BRCA2 mutations. We now know that other ethnic and geographic populations such as French Canadian individuals and certain populations from European decent may be at risk for these mutations. In terms of personal history of breast or ovarian cancer, we know that the type of cancer may be important. Women diagnosed with a so-called "Triple Negative Breast Cancer", may be at a significantly higher risk of carrying a mutation; that risk is estimated to range between $10 \%$ and $40 \%$. Also women with "HighGrade Serous Ovarian Cancer" (HGSOC), whether or not there is family history, the rationale is there is a high prevalence of mutations in BRCA (supported, however, by limited data), specifically in these women; these mutations do not have to be germline mutations, they can be somatic mutations, they can be changes within the BRCA gene itself or an epigenetic mutation.

Wider access to genetic testing is under evaluation. Professional societies do not recommend that children, even those with a family history suggestive of a harmful BRCA1 or BRCA2 mutation, undergo genetic testing for BRCA1 or BRCA2. No risk-reduction strategies exist for children, and children's risks of developing cancer type associated with a BRCA1 or BRCA2 mutation are extremely low. Genetic counseling is generally recommended before and after any genetic test for inherited cancer syndrome.A positive test result can bring relief from uncertainty and allow people to make informed decisions about their future, including taking steps to reduce their cancer risk. In addition, people who have a positive test result may be able to participate in medical research that could, in the long run, help reduce deaths from breast and ovarian cancer.

Several options are available for managing cancer risk in individuals who have a known harmful BRCA1 or BRCA2 mutation. These include enhanced screening, prophylactic (risk reducing) surgery, and chemoprevention. 


\section{Acknowledgments}

None.

\section{Conflicts of interest}

The authors have no financial conflicts of interest to declare.

\section{Funding}

None. 